\title{
Bouncing Universes in Scalar-Tensor Gravity Models admitting Negative Potentials
}

\author{
B. Boisseau $\stackrel{1 *}{\stackrel{1 *}{ } \text { H. Giacomini }{ }^{1 \dagger}, \text { D. Polarski }{ }^{2 \ddagger} ; \text { A. A. Starobinsky }}{ }^{3,4 \S}$ \\ ${ }^{1}$ Université de Tours, Laboratoire de Mathématiques et Physique Théorique, \\ CNRS/UMR 7350, 37200 Tours, France \\ ${ }^{2}$ Université Montpellier \& CNRS, Laboratoire Charles Coulomb, \\ UMR 5221, F-34095 Montpellier, France \\ ${ }^{3}$ L. D. Landau Institute for Theoretical Physics RAS, \\ Moscow 19334, Russian Federation \\ ${ }^{4}$ Kazan Federal University, Kazan 420008, \\ Republic of Tatarstan, Russian Federation
}

August 21, 2018

\begin{abstract}
We consider the possibility to produce a bouncing universe in the framework of scalar-tensor gravity models in which the scalar field potential may be negative, and even unbounded from below. We find a set of viable solutions with nonzero measure in the space of initial conditions passing a bounce, even in the presence of a radiation component, and approaching a constant gravitational coupling afterwards. Hence we have a model with a minimal modification of gravity in order to produce a bounce in the early universe with gravity tending dynamically to general relativity (GR) after the bounce.
\end{abstract}

PACS Numbers: 04.62.+v, 98.80.Cq

*email:bruno.boisseau@lmpt.univ-tours.fr

$\dagger$ email:hector.giacomini@lmpt.univ-tours.fr

‡email:david.polarski@umontpellier.fr

§email:alstar@landau.ac.ru 


\section{Introduction}

Since the realization that the strong curvature singularity arising in the past of our Universe in Friedmann-Lemaître-Robertson-Walker (FLRW) models may not be avoided in generic solutions of the Einstein gravity with matter in the form of an ideal fluid (only its type changes from an isotropic one to the generic BKL vacuum singularity), there were many attempts to construct solutions avoiding this singularity by having at least one bounce at some high value of curvature either using more complicated field-theoretical models of matter or by modifying gravity. The simplest example to obtain one bounce in a set of solutions having a nonzero measure in the space of initial conditions is presented by a massive scalar field in a closed (i.e. positively spatially curved) FLRW universe [1]. This does not remove the curvature singularity in a generic solution, even in the class of FLRW models, but shifts it to the past, beyond any given finite number of bounces of the scale factor. Non-singular FLRW solutions with an infinite number of bounces exist, too, but they are degenerate: they have a zero measure in the space of initial conditions [2], see also [3]. This occurs because a minimally coupled scalar field with a non-negative potential can violate the strong energy condition, but not the weak and null ones 1 However, having a bounce due to a positive spatial curvature requires severe fine tuning of initial conditions before the contraction stage in the past, see e.g. the calculations of the measure of those ones leading to a bounce in the above mentioned cosmological model in [1] and [6]. That is why it is much more interesting to obtain a non-degenerate bounce in FLRW models in the absence of spatial curvature. Previously known examples of such FLRW models are based on such radical modifications of general relativity (GR) as theories with scalar [7, 8] or tensor ghosts, loop quantum gravity (see e.g. [9]) or gravity described by an effectively non-local Lagrangian (see e.g.[10],[11], and [12] for a recent review). By contrast, in our paper we would like to restrict ourselves to the well-known and very modest modification of GR - scalar-tensor gravity without ghosts. On the other hand, we want to abandon the common assumption that the scalar field potential $V(\phi)$ in this gravity theory is nonnegative, we will even consider the case when it is unbounded from below. In Section 2, the bouncing model is presented and general equations and results are given. In Section 3 , the existence of physically viable bouncing solutions is shown and their behaviour is studied in details. Section 4 contains our conclusions.

\footnotetext{
${ }^{1}$ In the case of open (negatively spatially curved) FLRW models, a bouncing solution was found in 4 for a non-minimally coupled scalar field with a quartic potential (so this model represents a particular kind of scalar-tensor gravity models). However, later it was shown in [5] that this regular solution is degenerate, too: it is unstable against arbitrarily small anisotropic and/or inhomogeneous metric perturbations preventing transition to the regime with a tensor ghost (repulsive gravity).
} 


\section{A bouncing model}

Let us consider a universe where gravity is described by scalar-tensor theory. The Lagrangian density in the Jordan frame of the gravitational sector is given by

$$
L=\frac{1}{2}\left[F(\Phi) R-Z(\Phi) g^{\mu \nu} \partial \mu \Phi \partial \nu \Phi-2 U(\Phi)\right]
$$

Everywhere below we will use the freedom to take $Z=1$ or $Z=-1$, corresponding physically to $\omega_{B D}>0$ or $\omega_{B D}<0\left(\omega_{B D}=Z F\left(\frac{d F}{d \Phi}\right)^{-2}\right)$. For $\omega_{B D}<0$, the theory is ghost-free provided $-\frac{3}{2}<\omega_{B D}<0$. We consider further spatially flat FLRW universes with metric $d s^{2}=-d t^{2}+a^{2}(t) d \mathbf{x}^{2}$ yielding the following modified Friedmann equations

$$
\begin{aligned}
-3 F H^{2}+\frac{1}{2} Z \dot{\Phi}^{2}-3 H \dot{F}+U & =0, \\
2 F \dot{H}+Z \dot{\Phi}^{2}+\ddot{F}-H \dot{F} & =0 .
\end{aligned}
$$

with $H \equiv \frac{\dot{a}}{a}$. Here and below a dot, resp. a prime, stands for the derivative with respect to $t$, resp. to $\Phi$. Equations (2), (3) contain the equation of motion of $\Phi$

$$
Z(-\ddot{\Phi}-3 H \dot{\Phi})+3 F^{\prime}\left(\dot{H}+2 H^{2}\right)-U^{\prime}=0
$$

We start our analysis with $Z=1$ and we specialize to the following model

$$
\begin{aligned}
F & =-\frac{1}{6} \Phi^{2}+\kappa^{-2} \\
U & =\frac{\Lambda}{\kappa^{2}}-c \Phi^{4}
\end{aligned}
$$

where $\kappa^{-2}>0, \Lambda>0$ and $c$ are constant parameters, only $c$ being dimensionless. Surprisingly, while we arrived at this model by requiring that a combination of (2), (3) and (41), without derivation with respect to $t$, be zero, this model has been considered in the past in completely different contexts (see e.g. [13]). Thus (41) corresponds to a massless scalar field conformally coupled to Einstein gravity with an interaction potential $-c \Phi^{4}$. Therefore, the energy-momentum tensor of the field $\Phi$ is the sum of "dark" radiation with a negative energy density and a cosmological constant.

As a result, the system (2), (3) reduces to

$$
\begin{gathered}
3 H^{2}=\Lambda+\kappa^{2} \frac{A}{a^{4}} \\
\frac{1}{2}\left(\frac{d \chi}{d \eta}\right)^{2}-c \chi^{4}=A
\end{gathered}
$$

with an effective positive cosmological constant $\Lambda$ and the bare gravitational constant $8 \pi G=F^{-1}(\Phi=0) \equiv \kappa^{2}$, while $\chi=a \Phi$ and $\eta=\int d t / a(t)$. The crucial point is that the 
constant $A$ - the energy density of the field $\chi$ in Minkowski space-time - can be negative here.

For $A<0$, we have in front of us an explicit illustration of the possibility for scalartensor models to accommodate an effective dark energy component of the phantom type $\left(w_{\text {eff }}<-1\right)$ [14, 15] 2. Our system can be completely integrated. As we are interested in bouncing solutions, we must take $A<0$ and from (8), we have $c>0$ so that $U$ is necessarily an inverted potential, unbounded from below. In spite of looking unphysical at first sight, scalar fields with such an interaction have been often considered both in quantum field theory and cosmology, see e.g. [17].

Integrating (7), a bouncing solution is obtained

$$
\begin{aligned}
a & =a_{0} \cosh ^{\frac{1}{2}}\left[2 \sqrt{\frac{\Lambda}{3}} t\right], \\
H & =\sqrt{\frac{\Lambda}{3}} \tanh \left[2 \sqrt{\frac{\Lambda}{3}} t\right],
\end{aligned}
$$

where $a_{0}=\left(\frac{-A \kappa^{2}}{\Lambda}\right)^{\frac{1}{4}}$ is the value of $a$ at the bounce located at $t=0$ with a trivial redefinition of $t$. It satisfies $\dot{H}>0$ and has a constant Ricci scalar $R=6\left(\dot{H}+2 H^{2}\right)=4 \Lambda$. Integrating (8) the analytical expression for $\chi$ is obtained in terms of the Jacobi elliptic function $\operatorname{dn}(u \mid m)$ [18] as follows

$$
\chi(\eta)=-\left(\frac{-A}{c}\right)^{\frac{1}{4}} \frac{1}{\operatorname{dn}(u \mid 2)},
$$

with

$$
u=\operatorname{dn}^{-1}\left(-\left(\frac{\Lambda}{c \kappa^{2}}\right)^{\frac{1}{4}} \frac{1}{\Phi_{0}} \mid 2\right)+\sqrt{2}(-A c)^{\frac{1}{4}} \eta,
$$

where $\operatorname{dn}^{-1}(u \mid m)$ is the inverse function of $\operatorname{dn}(u \mid m)$ and $\Phi_{0}=\Phi(t=0)$. The conformal time $\eta(t)$, chosen here such that $\eta(0)=0$, is given by

$$
\eta(t)=-\frac{i}{a_{0} \sqrt{\frac{\Lambda}{3}}} F\left(i \sqrt{\frac{\Lambda}{3}} t \mid 2\right)
$$

where $F(x \mid m)$ is the elliptic integral of the first kind [18]. This function is odd and then $\eta(t)$ is real. It is possible to show that

$$
\eta(\infty)=\frac{1}{a_{0} \sqrt{\frac{2 \Lambda}{3}}} \frac{\Gamma^{2}\left(\frac{1}{4}\right)}{4 \sqrt{\pi}} .
$$

\footnotetext{
${ }^{2} \mathrm{~A}$ phenomenological dark energy model in GR based on (7) was considered in [16], leading to (9) and (10).
} 
So we get finally

$$
\Phi(t)=\frac{-\left(\frac{\Lambda}{c \kappa^{2}}\right)^{\frac{1}{4}}}{\sqrt{\cosh \left(2 \sqrt{\frac{\Lambda}{3}} t\right)}} \frac{1}{\operatorname{dn}\left(\operatorname{dn}^{-1}\left(-\left(\frac{\Lambda}{c \kappa^{2}}\right)^{\frac{1}{4}} \frac{1}{\Phi_{0}} \mid 2\right)-i \sqrt{2}\left(\frac{9 c}{\kappa^{2} \Lambda}\right)^{\frac{1}{4}} F\left(i \sqrt{\frac{\Lambda}{3}} t \mid 2\right) \mid 2\right)} .
$$

We note that under the transformation $Z \rightarrow-1, F \rightarrow-F, U \rightarrow-U$, the same solution (15) is obviously obtained and that (7) and (8) remain valid as well because (2), (3) and (41) are invariant under this transformation.

Another attractive feature of this model is the possibility to have a bounce in the presence of a true radiation component resulting in a trivial change $A \rightarrow A^{\prime}$ in (7). This can model a bounce in the primordial universe. The bounce will survive provided the sum of "dark" and true radiation remains negative or

$$
A+\rho_{\text {rad }, 0} a_{0}^{\prime 4} \equiv A^{\prime}=-\frac{\Lambda}{\kappa^{2}} a_{0}^{\prime 4}<0
$$

which shows that $\frac{A}{a_{0}^{4}}=\frac{A^{\prime}}{a_{0}^{\prime 4}}$ is invariant, the scale factor $a_{0}^{\prime}$ at the bounce decreases in the presence of radiation and also when $A$ increases towards zero. Let us finally note that the bounce disappears for $A^{\prime} \geq 0$.

\section{Study of the bouncing solution}

In spite of the fact that we have obtained an explicit closed form expression for $\Phi(t)$ we will study the behaviour of the bouncing solution by applying the qualitative theory of differential equations. This will provide us with a more intuitive and direct approach and will enable us to obtain results about the qualitative behaviour of our system otherwise difficult to find from (15). Solving for the wave equation recast into the form

$$
\ddot{\Phi}+3 H \dot{\Phi}+4 c \Phi\left(\tilde{\Phi}^{2}-\Phi^{2}\right)=0
$$

where $\tilde{\Phi} \equiv\left(\frac{\Lambda}{6 c}\right)^{\frac{1}{2}}$, and evaluating (2) at the bounce

$$
\frac{1}{2} \dot{\Phi}_{0}^{2}-c\left(\Phi_{0}^{4}-\Phi_{0, \min }^{4}\right)=0
$$

where $\Phi_{0, \min } \equiv\left(\frac{\Lambda}{\kappa^{2} c}\right)^{\frac{1}{4}}=\left(\frac{-A}{c a_{0}^{4}}\right)^{\frac{1}{4}}$, our system is automatically solved.

The inequality $\Phi^{2}<6 \kappa^{-2}$ is readily obtained from the requirement $F>0$ while $\Phi=0$ is impossible from (8) . Choosing $\Phi_{0}>0$, the following inequality is further obtained

$$
\tilde{\Phi}<\Phi_{0, \min } \leq \Phi_{0}<\Phi_{\max }
$$




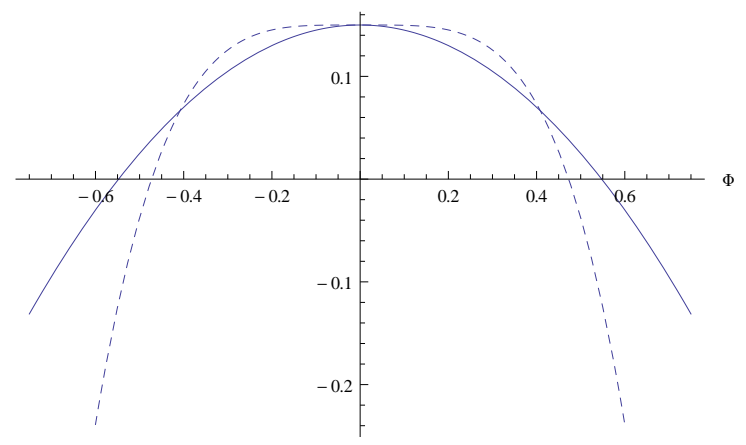

Figure 1: The functions $F$ (full) and $U$ (dashed) are displayed for the model parameters $\kappa^{2}=20, c=3$ and $\Lambda=3$. The two curves coincide at $\Phi=0$ because we have actually plotted $\Lambda F$. While $U$ vanishes at $\Phi \equiv \Phi_{0, \min }=\left(\frac{\Lambda}{\kappa^{2} c}\right)^{\frac{1}{4}}, F$ does so at $\Phi \equiv \Phi_{\max }=\frac{\sqrt{6}}{\kappa}$. For these parameters we have $\Phi_{0, \min }=0.4728, \Phi_{0, c r}=0.4936860, \Phi_{\max }=0.5477$. For $\Phi>0$, the range of possible values at the bounce lie in the interval $\Phi_{0, \min } \leq \Phi<\Phi_{\max }$ but only values in the interval $\Phi_{0, c r} \leq \Phi<\Phi_{\max }$ and with a negative slope will yield physically viable solutions.

with $6 \kappa^{-2} \equiv \Phi_{\text {max }}^{2}$. Indeed, from (21), the condition $U\left(\Phi_{0}\right) \equiv U_{0} \leq 0$ implies immediately $\Phi_{0} \geq \Phi_{0, \min }$, while $\Phi<\Phi_{\max }$, valid at all times, follows from the physical condition $F>0$. A non vanishing interval allowed for $\Phi_{0}$ requires $\Phi_{0, \min }<\Phi_{\max }$ or

$$
\frac{\Lambda \kappa^{2}}{36 c}<1
$$

implying also $\tilde{\Phi}<\Phi_{0, \min }$. From (18), with $\dot{\Phi}_{0}<0$ (the justification of this choice will be clear below), we obtain

$$
\dot{\Phi}_{0}=-\sqrt{2 c\left(\Phi_{0}^{4}-\Phi_{0, \text { min }}^{4}\right)},
$$

with $\dot{\Phi}_{0}$ decreasing when $\Phi_{0}$ is increasing.

We study the dynamics for $t \geq 0$. For $t<0$ the dynamics follows immediately due to the invariance of the system under reflection $t \rightarrow-t$. We will come back to this point at the end of our analysis. We will analyse now the possible existence of local maxima and minima for $\Phi(t)$. From (8) we readily obtain for $\dot{\Phi}=0$

$$
\Phi^{2}=\frac{\frac{1}{2} H^{2}+\sqrt{\frac{H^{4}}{4}-4 c A a^{-4}}}{2 c} .
$$

The choice of the positive sign before the square root is compulsory since $\Phi^{2}$ must be positive. It is straightforward to show that (20) implies $\Phi<\Phi_{\max }$ for $\Phi$ given by (22). Hence the inequality $\Phi<\Phi_{\max }$ follows when $\dot{\Phi}=0$. We have further from (3) when $\dot{\Phi}=0$ 


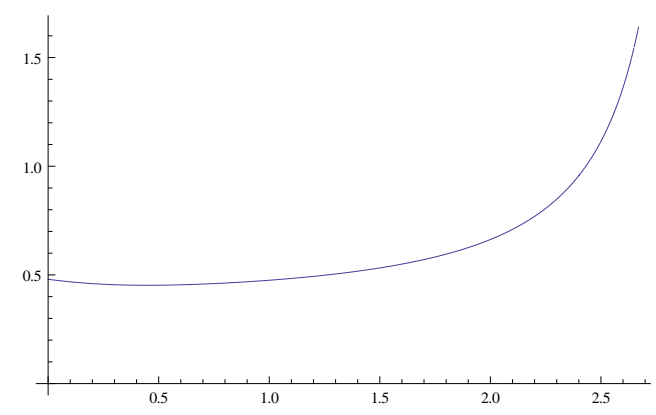

Figure 2: The function $\Phi(t)$ is shown starting at $t=0$ at the bounce for the same model parameters as in Figure 1. The initial value $\Phi_{0}$ is slightly below the critical value $\Phi_{0, c r}=$ $0.4936860 \ldots$ so this solution will diverge in a finite time.

that $-\Phi \ddot{\Phi}+6 F \dot{H}=0$, therefore $\ddot{\Phi}>0$. So we have shown the impossibility for $\Phi$ to have a maximum.

On the other hand if $\Phi$ has a finite limit for $t \rightarrow \infty$, we must have $\dot{\Phi}_{\infty}=0$ and $\ddot{\Phi}_{\infty}=0$, therefore (17) gives $4 c \Phi_{\infty}\left(\tilde{\Phi}^{2}-\Phi_{\infty}^{2}\right)=0$. Hence we have either $\Phi_{\infty}=0$ or $\Phi_{\infty}=\tilde{\Phi}$. These results imply in particular that had we chosen $\dot{\Phi}_{0}>0, \Phi$ would eventually enter the unphysical domain $\Phi \geq \Phi_{\max }$, so this choice is not allowed.

If the solution has a minimum, it is unique as $\Phi$ does not have any maximum. This minimum must be larger that $\tilde{\Phi}$ as a direct consequence of (17). Hence after the minimum, $\dot{\Phi}>0$ forever since there is no maximum and then $\Phi$ goes to infinity - therefore crossing the value $\Phi_{\max }$ - because the only possible finite limits $\Phi_{\infty}$ are lower than the minimum and cannot be reached. We will now show that in this case $\Phi$ tends to infinity in a finite time $t_{\infty}$. When $\Phi \rightarrow \infty$, keeping the leading terms in (2) we easily find $\dot{\Phi} \sim \sqrt{2 c} \Phi^{2}$, and after integration

$$
\Phi \sim \frac{1}{-\sqrt{2 c} t+k}
$$

with $k>0$ and $t_{\infty}=\frac{k}{\sqrt{2 c}}$. As a consequence $F$ becomes zero in a finite time too, rendering this solution unphysical. When $\Phi_{0}=\Phi_{0, \min }\left(U_{0}=0\right)$, the bounce is a minimum from eqs.(17) and then $\Phi$ goes to infinity in a finite time (see Figure 2).

Let us consider now the case where there is no minimum, i.e. $\dot{\Phi}<0$ for $t \geq 0$. From (17) we get $\ddot{\Phi}=-3 H \dot{\Phi}-4 c \Phi\left(\tilde{\Phi}^{2}-\Phi^{2}\right)>0$ as long as $\Phi>\tilde{\Phi}$. However when $\Phi(t)<\tilde{\Phi}$, the sign of $\ddot{\Phi}(t)$ can change. Two subcases will therefore arise:

1) $\Phi(t)$ is always greater than $\tilde{\Phi}$ and, since $\dot{\Phi}(t)<0$, tends asymptotically to $\Phi=\tilde{\Phi}$ with positive concavity $(\ddot{\Phi}(t)>0)$.

2) $\Phi(t)$ crosses $\tilde{\Phi}$ and, since $\dot{\Phi}(t)<0$, tends asymptotically to 0 . Below $\tilde{\Phi}, \ddot{\Phi}(t)$ can however change sign. 
In order to differentiate these two possibilities, we study the system in the neighborhood of $t=\infty$ where (17) can be recast as an autonomous planar system, viz.

$$
\begin{aligned}
& \dot{y_{1}}=y_{2}, \\
& \dot{y_{2}}=-\sqrt{3 \Lambda} y_{2}-2 \frac{\Lambda}{3} y_{1}+4 c y_{1}^{3},
\end{aligned}
$$

with $y_{1} \equiv \Phi$. This system has two hyperbolic fixed (critical) points: $(0,0)$ and $(\tilde{\Phi}, 0)$. The eigenvalues associated to $(0,0)$ are both negative, $\lambda_{1}=-\sqrt{\frac{\Lambda}{3}}$ and $\lambda_{2}=-\sqrt{\frac{4 \Lambda}{3}}$, so it is a stable node. The eigenvalues associated with $(\tilde{\Phi}, 0)$ have opposite sign, $\lambda_{1}=\sqrt{\frac{\Lambda}{3}}$ and $\lambda_{2}=-2 \sqrt{\frac{4 \Lambda}{3}}$, hence it is a saddle point. So the solution $\Phi \rightarrow \tilde{\Phi}$ is "unstable" and only with an exact initial value $\Phi_{0}$ can one reach the fixed point $\left(\Phi_{\infty}, \dot{\Phi}_{\infty}\right)=(\tilde{\Phi}, 0)$. In contrast, once we find some $\Phi_{0}$ value which yields a solution tending to 0 , we can vary $\Phi_{0}$ in a neighbourhood of this value and still go to the fixed point $(0,0)$.

By continuity, if we take $\Phi_{0}$ slightly larger than $\Phi_{0, \min }, \Phi(t)$ will still go to infinity in a finite time. Further increasing $\Phi_{0}$, two possibilities arise:

a) For $\Phi_{0, \text { min }} \leq \Phi_{0}<\Phi_{0, c r}$, where $\Phi_{0, c r}$ is some critical value, $\Phi(t)$ diverges in a finite time.

b) For $\Phi_{0, c r}<\Phi_{0}<\Phi_{\max }$ solutions will tend to zero.

In the absence of $\Phi_{0, c r}$ only the first behaviour holds. For the specific case $\Phi_{0}=\Phi_{0, c r}$, the solution will tend to $\tilde{\Phi}$. So this solution which tends to the saddle point is the separatrix which separates the two types of behaviours. Hence for given parameters $\kappa, \Lambda$ and $c$ satisfying (19), the two types of behaviours are possible provided the existence of the separatrix.

Determination of the initial value $\Phi_{0, c r}$ yielding the separatrix is a well-known problem in the theory of ordinary differential equations, known as the connexion problem [19]. For given values of the parameters of the system, it is not possible in general to determine analytically whether $\Phi_{0, c r}$ exists, neither to find a closed form expression for it when it does exist. We were able to find numerically $\Phi_{0, c r}$ for certain values of the parameters. For instance, for $\kappa^{2}=20, c=3$ and $\Lambda=3$, we find $\Phi_{0, c r}=0.4936860 \ldots$. An example of viable solutions is shown on Figure 3 .

Using the analytical results given earlier, one can derive an analytical expression for $\Phi_{0, c r}$

$$
\Phi_{0, c r}=-\left(\frac{\Lambda}{c \kappa^{2}}\right)^{\frac{1}{4}} \frac{1}{\operatorname{dn}\left(\left(\frac{2 j+1}{\sqrt{2}}-\left(\frac{9 c}{\Lambda \kappa^{2}}\right)^{\frac{1}{4}}\right) \frac{\Gamma^{2}\left(\frac{1}{4}\right)}{4 \sqrt{\pi}} \mid 2\right)} .
$$

Let us explain how (26) is obtained. As we look for the initial value $\Phi_{0, c r}$ for which $\Phi \rightarrow \tilde{\Phi}$, it is clear from (15) that $u(t=\infty)$ must then correspond to a zero of the function $\operatorname{dn}(u \mid 2)$, 

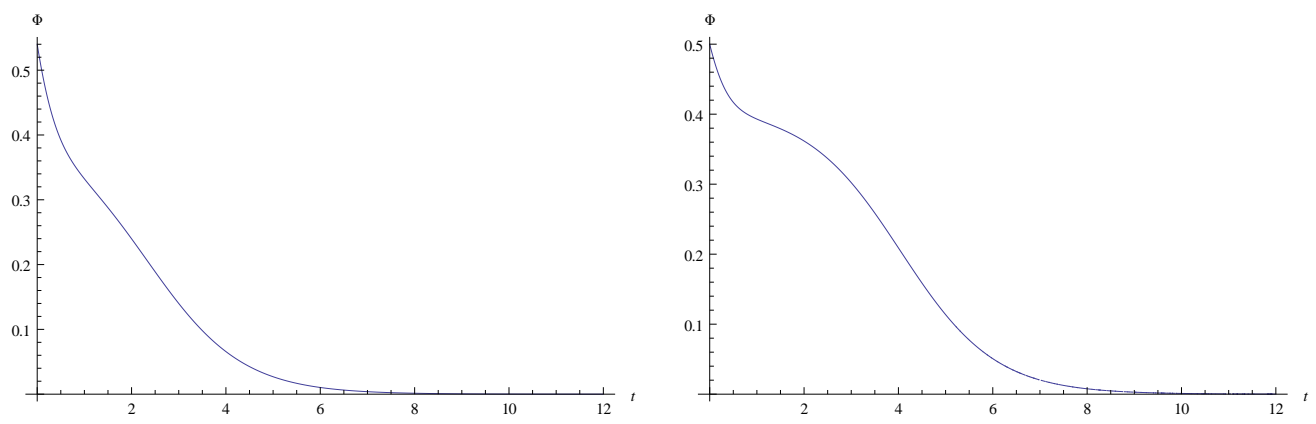

Figure 3: The function $\Phi(t)$ is shown starting at $t=0$ at the bounce for the same model parameters as in Figure 1. On the left panel, the initial value is $\Phi_{0}=0.54$ while on the right panel $\Phi_{0}=0.50$. As explained in the text $\ddot{\Phi}$ is positive above $\tilde{\Phi}$ whose value here is $\tilde{\Phi}=0.40824829 \ldots$, but can change sign below $\tilde{\Phi}$. The curve on the right has a more pronounced feature because it starts closer to $\Phi_{0, c r}=0.4936860 \ldots$ Both curves tend asymptotically to zero and constitute viable bouncing solutions. The curve starting precisely at $\Phi_{0, c r}$ will tend asymptotically to $\tilde{\Phi}$.

which are given by

$$
\frac{(2 j+1) \Gamma^{2}\left(\frac{1}{4}\right)}{4 \sqrt{2 \pi}}, \quad j=0,1, \ldots .
$$

Using (14), we obtain

$$
\frac{(2 j+1) \Gamma^{2}\left(\frac{1}{4}\right)}{4 \sqrt{2 \pi}}=\operatorname{dn}^{-1}\left(-\left(\frac{\Lambda}{c \kappa^{2}}\right)^{\frac{1}{4}} \frac{1}{\Phi_{0, c r}} \mid 2\right)+\left(\frac{c}{\Lambda \kappa^{2}}\right)^{\frac{1}{4}} \sqrt{3} \frac{\Gamma^{2}\left(\frac{1}{4}\right)}{4 \sqrt{\pi}} .
$$

Inverting this expression we obtain (26).

The value of $j$ must be chosen in such a way that $\Phi_{0, c r}$ lies inside the allowed interval (19) and that (28) is satisfied. As the function $\operatorname{dn}(u \mid 2)$ is periodic, only a small number of values of $j$ must be considered. The numerical value of $\Phi_{0, c r}$ given above was obtained from (26) for $j=1$. For some values of the model parameters and any value of $j, \Phi_{0, c r}$ is not in the allowed interval and then the resulting model is not viable.

In the presence of radiation one checks easily that the allowed range of initial values shrinks with a new larger minimal value $\Phi_{0, \min }^{\prime}$ satisfying

$$
\Phi_{0, \text { min }}^{\prime 4}=\Phi_{0, \text { min }}^{4} \frac{A}{A^{\prime}} .
$$

We have of course $A<A^{\prime}<0$. The quantities $\tilde{\Phi}, \Phi_{\max }$ on the other hand depend on the model parameters and remain unchanged. One can just repeat the proof of the existence of viable bouncing solutions. Hence, as long as we have $\Phi_{0, \min }^{\prime}<\Phi_{0, c r}$, the set of viable bouncing solutions remains. In the new expression for $\Phi(t)$ we will have 
$u \rightarrow \operatorname{dn}^{-1}\left(-\left(\frac{A}{A^{\prime}} \frac{\Lambda}{c \kappa^{2}}\right)^{\frac{1}{4}} \frac{1}{\Phi_{0}} \mid 2\right)-i \sqrt{2}\left(\frac{A}{A^{\prime}} \frac{9 c}{\kappa^{2} \Lambda}\right)^{\frac{1}{4}} F\left(i \sqrt{\frac{\Lambda}{3}} t \mid 2\right)$ while the same corrective factor $\left(\frac{A}{A^{\prime}}\right)^{\frac{1}{4}}$ appears in front of (15) as well. We have the obvious corresponding change in (28) and (26).

Let us consider next the possibility to have a viable bounce when $Z=-1$ in the absence of radiation. If we want to keep the conformal invariance, the absence of ghosts $\left(\omega_{B D}>-\frac{3}{2}\right)$ and the existence of a bounce, we are led to the following ansatz:

$$
F_{Z=-1}(\Phi)=-F_{Z=1}(\Phi) \quad U_{Z=-1}(\Phi)=-U_{Z=1}(\Phi)
$$

with the parameters $\kappa^{-2}, \Lambda$ still positive. The quantities $F_{Z=1}$, resp. $U_{Z=1}$, correspond to (5), resp. (6). It is easily checked that (7) is retrieved leading to a bounce provided the quantity $A$ is negative. This implies here too that $c>0$, but now $U_{Z=-1}$ no longer represents an inverted potential and we have $U_{Z=-1}(\Phi=0)<0$. As we have emphasized earlier, with (30) the same equation of motion for the field $\Phi$ is obtained therefore leading to the same solution (15). However, the crucial difference is that the domain of physical validity differs in both cases: now all values $\Phi>\frac{\sqrt{6}}{\kappa}$ satisfy $F_{Z=-1}>0$. At the bounce, it is seen from (2) that the potential must satisfy $U_{Z=-1}\left(\Phi_{0}\right) \geq 0$ yielding in this case too $\Phi_{0} \geq \Phi_{0, \text { min }}$.

Up to these differences, we can essentially repeat the analysis performed for $Z=1$. It is easy to show using (4) that $\dot{\Phi}=0$ implies $\ddot{\Phi}>0$ for $\Phi>\tilde{\Phi}$, and $\ddot{\Phi}<0$ for $\Phi<\tilde{\Phi}$. On the other hand from (3),$\dot{\Phi}=0$ implies $\ddot{\Phi}<0$. Hence $\dot{\Phi}=0$ is not possible for $\Phi>\tilde{\Phi}$ while it would correspond to a maximum for $\Phi<\tilde{\Phi}$. On the other hand, from (4) only $\tilde{\Phi}$ remains an acceptable finite limit of $\Phi$ because $\Phi=0$ is now excluded. Imposing the condition $\frac{\Lambda \kappa^{2}}{36 c}>1$, we now obtain

$$
\frac{\sqrt{6}}{\kappa}<\Phi_{0, \min }<\tilde{\Phi}
$$

If $\Phi_{0}>\tilde{\Phi}, \dot{\Phi}_{0}>0$ implies $\Phi \rightarrow \infty$ while $\dot{\Phi}_{0}<0$ can in principle produce two possible behaviours namely $\Phi \rightarrow \tilde{\Phi}$ (in that case $\Phi_{0}=\Phi_{0, c r}$ ) or $\Phi \rightarrow 0$, the latter being unviable. The first possibility cannot occur. Indeed with $\dot{\Phi}_{0}<0$, some initial values above as well as below $\Phi_{0, c r}$ would tend to zero. But the particular value $\Phi_{0, c r}$ must separate two different behaviours, hence this is not possible. When $\Phi_{0}<\tilde{\Phi}, \Phi \rightarrow 0$ for a negative initial slope because there is no minimum. With $\dot{\Phi}_{0}>0$, three different behaviours can be found: $\Phi \rightarrow 0$ when $\Phi$ goes through a maximum (below $\tilde{\Phi}$ ), $\Phi \rightarrow \infty$ when there is no maximum and finally $\Phi \rightarrow \tilde{\Phi}$ for $\Phi_{0, c r}$ which now separates the two behaviours mentioned earlier, its existence is therefore allowed in this case. It is interesting that while a viable bounce can exist for $Z=-1$ with a potential bounded from below, it has measure zero in the initial conditions, only one particular set of initial conditions at the bounce is acceptable. The expression of $\Phi_{0, c r}$ for this case is also given by (26) . 


\section{Conclusions}

We have shown that for $Z=1$ only three types of solutions are possible. All stable solutions vanishing asymptotically are especially interesting, their initial value $\Phi_{0}$ at the bounce can vary in some range $\Phi_{0, c r}<\Phi_{0}<\sqrt{6} \kappa^{-1}$. The solution with $\Phi_{0}=\Phi_{0, c r}$ is unique (measure zero) and moreover unstable. The stable solutions lead to the General Relativity limit if we assign to the bare gravitational constant $\kappa^{2}$ its numerical value in GR. Consistency of our model requires then the inequality $\frac{\Lambda \kappa^{2}}{8 \pi} \ll 1$. If we want to embed our model in a realistic cosmology, this attractive feature is even reinforced by the possibility to add a radiation component to our bouncing model.

Further, due to the invariance of the equations under the change $t \rightarrow-t$, the solutions for $t<0$ are obtained from those found for $t>0$ changing the sign of $\dot{\Phi}$. Hence, regular solutions for $t>0$ will diverge in the past both for $Z=1$ and $Z=-1$. Still, starting with regular initial values at $-\infty<t<0$, it is possible to have a bouncing universe regular in the future. Note that a solution symmetric around the bounce requires $\dot{\Phi}_{0}=0$ which will diverge both in the past and in the future.

It is well known that a bounce in the early universe can cure many of the problems occurring in Big Bang cosmology besides of course avoiding the Big Bang singularity itself. Without exploring further this scenario, we have shown that surprisingly such a bounce can occur for a FLRW flat universe within one of the oldest and best understood alternatives to GR in a model tending dynamically to GR after the bounce. The key point is to admit a negative scalar field potential that can lead to a transient negative energy density of the scalar field for some range of parameters 3 Of course, to make the model cosmologically viable, one has to generalize it somehow to open channels for decay of the effective positive (and large) cosmological constant $\Lambda$ into other quantum fields including those of the Standard Model of elementary particles at late times.

\section{Acknowledgments}

A.S. was partially supported by the RFBR Grant No. 14-02-00894 and by the Russian Government Program of Competitive Growth of the Kazan Federal University.

\footnotetext{
${ }^{3}$ In this connection, the early paper [20] has to be mentioned, too, where a FLRW bounce in $f(R)$ gravity was achieved in case of the scalar degree of freedom in this theory (scalaron) being tachyonic permanently that corresponds to a negative (and even unbounded from below) effective scalar field potential in the Einstein frame, see also [21].
} 


\section{References}

[1] A. A. Starobinsky, Sov. Astron. Lett. 4, 82 (1978).

[2] D. N. Page, Class. Quantum Grav. 1, 417 (1984).

[3] A. Yu. Kamenshchik, I. M. Khalatnikov, A. V. Toporensky, Int. J. Mod. Phys. D7, 673 (1997) [gr-qc/9801064].

[4] V. N. Melnikov and S. V. Orlov, Phys. Lett. A 70, 263 (1979).

[5] A. A. Starobinsky, Sov. Astron. Lett. 7, 36 (1981).

[6] G. W. Gibbons and N. Turok, Phys. Rev. D 77, 063516 (2008) [hep-th/0609095].

[7] D. Tretyakova, A. Shatskij, I. Novikov and S. Alexeyev, Phys. Rev. D 85, 124059 (2012) arXiv:1112.3770.

[8] J. D. Barrow and D. Sloan, Phys. Rev. D 88, 023518 (2013) arXiv:1304.6699].

[9] A. Ashtekar, T. Pawlowski, P. Singh, Phys. Rev. D 74, 084003 (2006) [gr-qc/0607039].

[10] T. Qiu, J. Evslin, Y. F. Cai, M. Li and X. Zhang, JCAP 1110, 036 (2011) arXiv:1108.0593.

[11] D. A. Easson, I. Sawicki, A. Vikman, JCAP 1111, 021 (2011) arXiv:1109.1047.

[12] D. Battefeld, P. Peter, Phys. Rept. 571, 1 (2015) arXiv:1406.2790].

[13] C. Martinez, R. Troncoso, J. Zanelli, Phys. Rev. D67, 024008 (2003) hep-th/0205319].

[14] B. Boisseau, G. Esposito-Farèse, D. Polarski and A. A. Starobinsky, Phys. Rev. Lett. 85, 2236 (2000) gr-qc/0001066.

[15] R. Gannouji, D. Polarski, A. Ranquet, A. A. Starobinsky, JCAP 0609, 016 (2006) astro-ph/0606287.

[16] D. Polarski, arXiv:1303.4470].

[17] V. A. Rubakov, JCAP 0909, 030 (2009) arXiv:0906.3693.

[18] M. Abramowitz and I. A. Stegun, Handbook of mathematical functions with formulas, graphs, and mathematical tables (Milton Abramowitz and Irene A. Stegun, eds.), Dover Publ., Inc., New York, 1992 (reprint of the 1972 edition).

[19] B. Boisseau, P. Forgacs and H. Giacomini, J. Phys. A40, F215 (2007) hep-th/0611306]. 
[20] T. V. Ruzmaikina and A. A. Ruzmaikin, Sov. Phys. - JETP 30, 372 (1970).

[21] M. M. Ivanov and A. V. Toporensky, Int. J. Mod. Phys. D 21, 1250051 (2012) arXiv:1112.4194. 